\title{
Immunocytochemical reactions in the brittle-star: ophiocomina nigra after immunization
}

\begin{abstract}
Immunizations of Ophiocomina nigra were performed with either Bovine Serum Albumine (BSA) or Horse-Radish Peroxydase (HRP) in aquarium containing running sea water. An immunocytochemical test was done in presence of HRP, diaminobenzidin, $\mathrm{H}_{2} \mathrm{O}_{2}$ for both animals: Control ones, BSA ones and HRP ones. Positive reactions only occur in injected animals to HRP. So the brittle star O.nigra as the sea star: Asterina gibbosa discriminates HRP from BSA: it is an antibody-like reaction. Plasmolymphocytic cells were evoked.
\end{abstract}

Keywords: ophiocomina nigra, bsa, hrp, axial organ, asterina gibbosa
Volume 2 Issue 6 - 2017

\author{
Michel Leclerc Henrik Sundh,' Bodil \\ Hernroth ${ }^{2}$ \\ 'Department of Biological and Environmental Sciences, \\ Gothenburg University, Sweden \\ ${ }^{2}$ Christianstad University, Sweden
}

Correspondence: Michel Leclerc Henrik Sundh, 556 rue Isabelle Romée, 45640 Sandillon, France, Emailmleclerc45@gmail.com

Received: June 12, 2017| Published: June 21, 2017

\section{Introduction}

All echinoderms (Echinodermata), except holothurids, possess an axial organ (AO) as part of their coelomic and haemal system. Immunocytochemical positive reactions were observed in 1973 in the sea star Asterina gibbosa (Asterid, Echinoderm) after injections of various proteins. ${ }^{1}$ It seemed interesting to look for similar reactions in the Ophuirid: Ophiocomina nigra (another echinoderm) which possesses also an axial organ. The sea star axial organ, an ancestral lymphoïd organ shows immune adaptative reactions ${ }^{2}$ and presents a primitive antibody ${ }^{3}$ The question was: «Are the Ophiocomina and Asterina gibbosa axial organs similars from the point of view of immunocompetence ?» So, we have repeated an experimental protocol in Ophiocomina nigra which resembles to the sea star one of 1973 , at the level, exclusively, of cytologic observations.

\section{Materials and methods}

Ophiocomina nigra were obtained from the Marine Biological Institute of Gothenburg and placed in aquarium with running sea water. 20 animals were put in three parts:

I. Control ones (6 animals): first aquarium

II. BSA (Bovine serum albumin) ones (7 animals): $2 \mathrm{~d}$ aquarium

III. HRP (Horse-radish peroxydase) ones ( 7 animals): $3 \mathrm{~d}$ aquarium

Control animals were not immunized. On the other hand BSA animals received at time $\mathrm{t}=010 \mu \mathrm{l}$ of $\mathrm{BSA}(1 \mathrm{mg} / \mathrm{ml}$ solution $)$ and at time $\mathrm{t}=7$ days after, again, $10 \mu 1$ of BSA( same concentration). HRP animals received $10 \mu 1$ of HRP (Sigma Products) of a solution at $1 \mathrm{mg} /$ $\mathrm{ml}$ at times similar as those of BSA animals then a second injection of $10 \mu 1$ of HRP. 3days after the last injection. All animals were sacrifiied and axial organs (A.O) were excised. Fixation of A.O in glutaraldehyde occured ( $2 \%$ in cacodylate buffer). Then A.O were washed in buffer. Incubation in HRP $(1 \mathrm{mg} / \mathrm{ml})$ was performed for all A.O. Washing in buffer was following. Incubation in diaminobenzidin $(1 \mathrm{mg} / \mathrm{ml})$ and $\mathrm{H}_{2} \mathrm{O}_{2}$ (1 drop) was realized then washing in buffer occured $\mathrm{O} / \mathrm{N}$. We did dehydratation in alcohol and then embedding in paraffin. Sectioning was made at $4 \mu$. We used Heathrow scientific LLC slides. Coloration was performed with Giemsa (1min). Observations were done with a microscope Zeiss.

\section{Results}

Briefly we observe neither labelling in control animals, nor in BSA treated animals. In immunized animals to HRP a positive labelling(color dark-brown) occurs in hyaloplasm in ergastoplasm and sometimes in the perinuclear space (Figures $1 \& 2$ ). Labelling is about $5 \%$ of the whole cell population. It is of $0 \%$ for BSA treated animals (Figure 3). Plasmolymphocytoplasmic and lymphocytes cells seem to exist in Ophiocomina nigra as for Asterina gibbosa but it must be corroborated by TEM observations. ${ }^{1}$

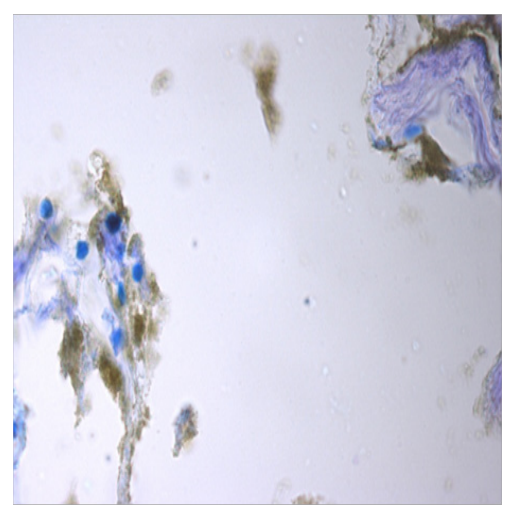

Figure I HRP (Magnification 1000).

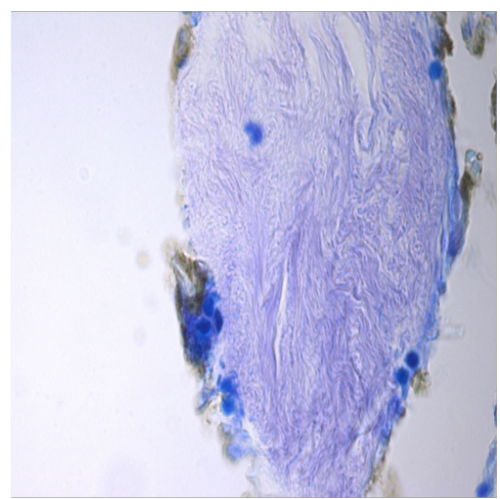

Figure 2 HRP (Magnification 100). 


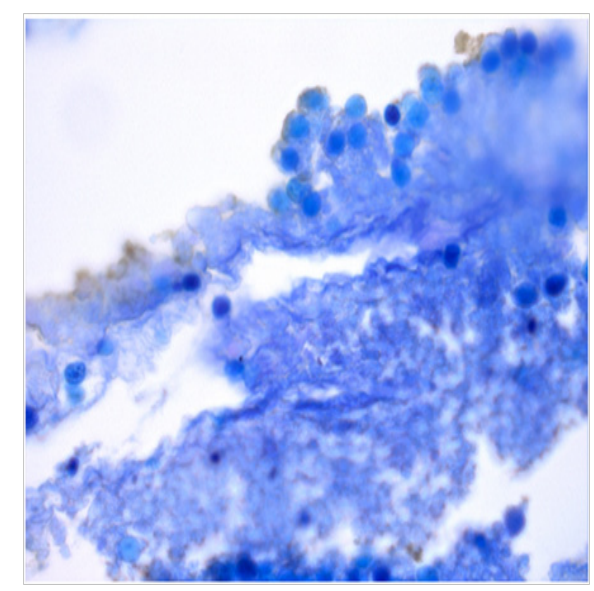

Figure 3 BSA (Magnification 1000).

\section{Discussion and conclusion}

It is shown that antigen-antibody like reactions occur in the brittle star: Ophiocomina nigra. It opens an immense field of experiments we may compare to those of Asterids (another echinoderm): i-e research of lymphocytes (like sea star B and T lymphocytes) Research of Primitive Brittle star antibody, and brittle star genomic studies. ${ }^{4}$ In fact, It would be interesting to study the genome of O.nigra and to compare the sea star immune genes to brittle star ones. We hope that the present work will help in suggesting provocative and imaginative ideas on adaptative immunity in marine invertebrates.

\section{Acknowledgements}

None.

\section{Conflict of interest}

The author declares no conflict of interest.

\section{References}

1. Leclerc M. Ann Immunol. 1973;124C:363-374.

2. Leclerc M, Brillouet C. Evidence of antibody-like substances secreted by axial organ cells of the starfish Asterias rubens. Immunol Lett. 1981;2(5-6):279-281.

3. Leclerc M. Amer J munol. 2012;8(3):78-83.

4. Vincent N, Osteras M, Otten P, et al. A new gene in A. rubens: A sea star Ig kappa gene. Meta Gene. 2014;2:320-322. 